\title{
Bayesian Analysis of Realized Matrix-Exponential GARCH Models
}

Instituto

Complutense de Análisis Económico

\section{Manabu Asai}

Faculty of Economics Soka University, Japan

\section{Michael McAleer}

Department of Finance Asia University, Taiwan and

Discipline of Business Analytics University of Sydney Business School, Australia and Econometric Institute Erasmus School of Economics

Erasmus University Rotterdam, The Netherlands and

Department of Quantitative Economics

Complutense University of Madrid, Spain and

Institute of Advanced Sciences

Yokohama National University, Japan

\begin{abstract}
The paper develops a new realized matrix-exponential GARCH (MEGARCH) model, which uses the information of returns and realized measure of co-volatility matrix simultaneously. The paper also considers an alternative multivariate asymmetric function to develop news impact curves. We consider Bayesian MCMC estimation to allow non-normal posterior distributions. For three US financial assets, we compare the realized MEGARCH models with existing multivariate GARCH class models. The empirical results indicate that the realized MEGARCH models outperform the other models regarding in-sample and out-of-sample performance. The news impact curves based on the posterior densities provide reasonable results.
\end{abstract}

Keywords Multivariate GARCH; Realized Measure; Matrix-Exponential; Bayesian Markov chain Monte Carlo method; Asymmetry.
JEL Classification
C11, C32.

\section{Working Paper no 1804 \\ January, 2018}




\title{
Bayesian Analysis of Realized Matrix-Exponential GARCH Models*
}

\author{
Manabu Asai \\ Faculty of Economics \\ Soka University, Japan \\ Michael McAleer \\ Department of Finance \\ Asia University, Taiwan \\ and \\ Discipline of Business Analytics \\ University of Sydney Business School, Australia \\ and \\ Econometric Institute \\ Erasmus School of Economics \\ Erasmus University Rotterdam, The Netherlands \\ and \\ Department of Quantitative Economics \\ Complutense University of Madrid, Spain \\ and \\ Institute of Advanced Sciences \\ Yokohama National University, Japan
}

January 2018

\footnotetext{
${ }^{*}$ The authors are most grateful to Yoshi Baba and Thomas Leonard for very helpful comments and suggestions. The first author acknowledges the financial support of the Japan Ministry of Education, Culture, Sports, Science and Technology, Japan Society for the Promotion of Science, and Australian Academy of Science. The second author is most grateful for the financial support of the Australian Research Council, National Science Council, Ministry of Science and Technology (MOST), Taiwan, and the Japan Society for the Promotion of Science. Address for correspondence: Faculty of Economics, Soka University, 1-236 Tangi-machi, Hachioji, Tokyo 192-8577, Japan. Email address: m-asai@soka.ac.jp.
} 


\begin{abstract}
The paper develops a new realized matrix-exponential GARCH (MEGARCH) model, which uses the information of returns and realized measure of co-volatility matrix simultaneously. The paper also considers an alternative multivariate asymmetric function to develop news impact curves. We consider Bayesian MCMC estimation to allow non-normal posterior distributions. For three US financial assets, we compare the realized MEGARCH models with existing multivariate GARCH class models. The empirical results indicate that the realized MEGARCH models outperform the other models regarding in-sample and out-of-sample performance. The news impact curves based on the posterior densities provide reasonable results.
\end{abstract}

Keywords: Multivariate GARCH; Realized Measure; Matrix-Exponential; Bayesian Markov chain Monte Carlo method; Asymmetry.

JEL Classification: C11, C32. 


\section{Introduction}

Estimation and forecasting time-varying co-volatilities between assets plays an important role in asset pricing, portfolio selections, and risk management. A popular approach is to estimate conditional covariance matrices via the multivariate models of the class of generalized autoregressive conditional heteroskedasticity (GARCH) (see the survey paper by McAleer (2005) as an example).

Over the past two decades, realized measures of volatility have received unprecedented attention in the academic literature on modeling and forecasting of stock market returns volatility. In the traditional literature on GARCH models, Engle and Gallo (2006) and Shephard and Sheppard (2010), among others, incorporated realized measures for modeling and forecasting volatility. In addition, Hansen, Huang, and Shek (2012) suggested the realized GARCH framework, which provides a structure for the joint modeling of returns and realized measures of volatility. By extending the work of Hansen, Huang, and Shek (2012), Hansen and Huang (2016) developed the realized exponential GARCH (EGARCH) model, which is an extension of the EGARCH model of Nelson (1991).

In this paper, we consider a multivariate extension of the realized EGARCH model of Hansen and Huang (2016). For the model specification, two features of the EGARCH model of Nelson (1991) are to accommodate asymmetric effects and to guarantee the positive value of conditional volatility via the exponential transformation. For asymmetric effects from asset returns to future volatility, Hansen and Huang (2016) consider the second-order approximation of Hermite polynomials, rather than the absolute value function of standardized returns, as used in Nelson (1991). We consider a multivariate version of this type of specification, and develops news impact curves, as in Engle and $\mathrm{Ng}$ (1993). Turning to the positive definiteness of conditional co-volatility, we use the matrix-exponential transformation as in Chiu, Leonard, and Tsui (1996) and Kawakatsu 
(2005). We also incorporate measurement errors of realized volatility and co-volatility measures in the specification of the new realized matrix-exponential GARCH model.

For estimating the multivariate GARCH models, Vrontos et al. (2003) estimated several bivariate $\mathrm{ARCH}$ and GARCH models, and found that maximum likelihood (ML) estimates of the parameters were different from their Bayesian MCMC estimates. As the differences can be caused by the non-normality of the parameters, Vrontos et al. (2003) suggest careful interpretation of the ML estimation. For this reason, the Bayesian Markov chain Monte Carlo (MCMC) technique is considered in the paper, so that non-normal posterior distributions are obtained.

The remainder of the paper is organized as follows. Section 2 introduces the new realized MEGARCH model and its news impact curve. Section 3 explains the MCMC algorithm for the Bayesian estimation in detail. Section 4 provides an empirical example for three stocks traded on the New York Stock Exchange. Section 4 compares five kinds of symmetric and asymmetric models, using the deviance information criterion of Spiegelhalter et al. (2002) and the Frovenius norm of forecast error. Section 4 also examines the MCMC estimates of the parameters, and presents the news impact curves for describing effects from stock returns to future (co-)volatility of its own and other assets.. Finally, Section 5 gives some concluding remarks.

\section{Realized Matrix-Exponential GARCH Model}

We develop a multivariate extension of the realized EGARCH model of Hansen and Huang (2016). Let $y_{t}$ and $x_{t}$ be a financial asset return at day $t$ and the log of its realized volatility measure, 
respectively. The Realized EGARCH of Hansen and Huang (2016) is given by:

$$
\begin{aligned}
y_{t} & =\varepsilon_{t} \exp \left(h_{t} / 2\right), \quad \varepsilon_{t} \sim N(0,1) \\
x_{t} & =\xi+h_{t}+\delta\left(\varepsilon_{t}\right)+u_{t}, \quad u_{t} \sim N\left(0, \sigma_{u}^{2}\right) \\
h_{t+1} & =\omega+\phi\left(h_{t}-\omega\right)+\tau\left(\varepsilon_{t}\right)+\psi u_{t},
\end{aligned}
$$

where $\delta(\varepsilon)=\delta_{1} \varepsilon+\delta_{2}\left(\varepsilon^{2}-1\right)$ and $\tau(\varepsilon)=\tau_{1} \varepsilon+\tau_{2}\left(\varepsilon^{2}-1\right)$, and $\varepsilon_{t}$ and $u_{t}$ follow independent normal distributions. The conditional log-volatility, $h_{t}$, is determined by past information of innovation terms, $\varepsilon_{t}$ and $u_{t}$. The asymmetric function, $\delta(\varepsilon)$ and $\tau(\varepsilon)$, can be interpreted as the secondorder approximation of the Hermite polynomials. Hansen and Huang (2016) consider a more general framework, but their empirical results indicate that the above specification is sufficient for practical purposes.

We extend the univariate model (1) to the multivariate case by considering matrix-exponential transformation, as in Chiu et al. (1996). Chiu et al. (1996) suggested using the matrix-exponential transformation in order to guarantee the positive definiteness of the covariance matrix. Kawakatsu (2006) developed matrix-exponential GARCH models. For any square matrix, $A$, the matrixexponential transformation is defined by $\operatorname{Exp}(X)=\sum_{i=0}^{\infty}(1 / i !) A^{i}$, with $A^{0}=I$. The same result is obtained by working with the spectral decomposition, as we have $\operatorname{Exp}(A)$ by replacing the eigenvalues by their exponential transformation. Note that $\operatorname{Exp}(A)$ is positive definite, whenever $A$ is symmetric. In the same manner, $\log (B)$ is defined by its spectral decomposition of a positive definite matrix, $B$, with replacement of the logarithmic transformation of eigenvalues.

Let $\boldsymbol{y}_{t}$ be an $m$-dimensional vector of financial asset returns. Define the matrix-logarithmic transformation of an $m \times m$ matrix of realized volatility measures as $\boldsymbol{X}_{t}$. We consider the realized 
matrix-exponential GARCH (MEGARCH) model defined by:

$$
\begin{aligned}
\boldsymbol{y}_{t} & =\operatorname{Exp}\left(\frac{1}{2} \boldsymbol{H}_{t}\right) \varepsilon_{t}, \quad \varepsilon_{t} \sim N\left(\mathbf{0}, \boldsymbol{I}_{m}\right) \\
\boldsymbol{X}_{t} & =\boldsymbol{\Xi}+\boldsymbol{H}_{t}+\boldsymbol{\Delta}\left(\varepsilon_{t}\right)+\boldsymbol{U}_{t}, \quad \boldsymbol{u}_{t}=\operatorname{vech}\left(\boldsymbol{U}_{t}\right) \sim N\left(\mathbf{0}, \boldsymbol{\Sigma}_{u}\right) \\
\boldsymbol{H}_{t+1} & =\boldsymbol{\Omega}+\boldsymbol{\Phi} \odot\left(\boldsymbol{H}_{t}-\boldsymbol{\Omega}\right)+\boldsymbol{G}\left(\varepsilon_{t}\right)+\boldsymbol{\Psi} \odot \boldsymbol{U}_{t},
\end{aligned}
$$

where $\boldsymbol{\Xi}, \boldsymbol{\Omega}, \boldsymbol{\Phi}, \boldsymbol{\Psi}$ are $m \times m$ symmetric matrices of parameters, $\boldsymbol{\Sigma}_{u}$ is an $m^{*}$-dimensional positive definite matrix with $m^{*}=m(m+1) / 2, \odot$ denotes the operator of element-wise multiplication (Hadamard product), $\varepsilon_{t}$ and $\boldsymbol{u}_{t}$ follow independent multivariate normal distributions, and $\boldsymbol{\Delta}(\boldsymbol{\varepsilon})$ and $\boldsymbol{G}(\boldsymbol{\varepsilon})$ are matrices of asymmetric functions, as defined below. By the specification (2)-(4), the conditional volatility matrix, $\operatorname{Exp}\left(\boldsymbol{H}_{t}\right)$, is determined by past information of innovation terms, $\varepsilon_{t}$ and $\boldsymbol{u}_{t}$.

As multivariate extensions of asymmetric functions, $\delta(\varepsilon)$ and $\tau(\varepsilon)$, we define:

$$
\begin{aligned}
& \Delta(\varepsilon)=\varepsilon \boldsymbol{\delta}^{\prime}+\boldsymbol{\delta} \varepsilon^{\prime}+\Upsilon \odot\left(\varepsilon \varepsilon^{\prime}-\boldsymbol{I}_{m}\right), \\
& \boldsymbol{G}(\varepsilon)=\varepsilon \gamma^{\prime}+\gamma \varepsilon^{\prime}+\Lambda \odot\left(\varepsilon \varepsilon^{\prime}-\boldsymbol{I}_{m}\right),
\end{aligned}
$$

respectively, where $\boldsymbol{\delta}$ and $\boldsymbol{\gamma}$ are $m$-vectors of parameters, and $\boldsymbol{\Upsilon}$ and $\Lambda$ are $m \times m$ symmetric matrices. By the specification (5), we obtain, $E\left[\boldsymbol{\Delta}\left(\varepsilon_{t}\right)\right]=\boldsymbol{O}$ and $E\left[\boldsymbol{\Delta}\left(\boldsymbol{G}_{t}\right)\right]=\boldsymbol{O}$ with finite covariance matrices.

By the structure of the realized MEGARCH model (2)-(5), $\boldsymbol{y}_{t}$ and $\boldsymbol{X}_{t}$ are stationary, if $\left\{\boldsymbol{H}_{t}\right\}$ is stationary. Since the process, $\left\{\boldsymbol{G}\left(\varepsilon_{t}\right)+\boldsymbol{\Psi} \odot \boldsymbol{U}_{t}\right\}$, has an independent and identical distribution with mean zero, it can be considered as an innovation term. Hence the stationary condition is $\left|\phi_{i j}\right|<1$, where $\phi_{i j}$ is the $(i, j)$-element of $\boldsymbol{\Phi}$.

We may consider an alternative specification of the asymmetric function based on the MEGARCH 
model of Kawakatasu (2006), as:

$$
\begin{aligned}
& \boldsymbol{\Delta}(\varepsilon)=\text { unvech }\left\{\boldsymbol{B}_{\delta} \varepsilon+\boldsymbol{F}_{\delta}\left(\varepsilon^{*}-E\left(\varepsilon^{*}\right)\right)\right\}, \\
& \boldsymbol{G}(\boldsymbol{\varepsilon})=\operatorname{unvech}\left\{\boldsymbol{B} \boldsymbol{\varepsilon}+\boldsymbol{F}\left(\boldsymbol{\varepsilon}^{*}-E\left(\varepsilon^{*}\right)\right)\right\},
\end{aligned}
$$

where $\boldsymbol{\varepsilon}^{*}$ is an $m$-vector with $i$ th element $\left|\varepsilon_{i}\right|$, unvech $(\cdot)$ is the inverse operator of vech, and $\boldsymbol{B}_{\delta}, \boldsymbol{F}_{\delta}$, $\boldsymbol{B}$, and $\boldsymbol{F}$ are the $m^{*} \times m$ matrices with $m^{*}=m(m+1) / 2$. These two realized MEGACRH models reduce to the MEGARCH model by omitting (3), and setting $\boldsymbol{\Psi}=\boldsymbol{O}$ in (4). In our empirical analysis for model comparisons, we use the two MEGARCH and two realized MEGARCH models, namely the MEGARCH model with (5) (quadratic function), the MEGARCH model with (6) (Kawakatsu, 2006), the Realized MEGARCH model with (2)-(5), and the realized MEGARCH model with (2)-(4) and (6) (Kawaktsu-type).

As an extension of Engle and Ng (1993), we can plot the news impact curve (NIC) via the function

$$
\boldsymbol{V}=\operatorname{Exp}\left(\boldsymbol{\Omega}+G\left(\underline{\boldsymbol{V}}^{-1 / 2} \boldsymbol{y}\right)\right)
$$

whih is obtained by setting $\boldsymbol{H}_{t}=\boldsymbol{\Omega}, \boldsymbol{U}_{t}=\boldsymbol{O}$, and $\operatorname{Exp}\left(\boldsymbol{H}_{t}\right)=\underline{\boldsymbol{V}}$ in the realized MEGARCH model, where $\underline{\boldsymbol{V}}=E\left(\boldsymbol{y}_{t} \boldsymbol{y}_{t}^{\prime}\right)$.

\section{Bayesian Estimation}

\subsection{MCMC Algorithm}

In this subsection, we describe the Markov chain Monte Carlo (MCMC) technique for estimating the realized MEGARCH model. For this purpose, we review briefly the development of MCMC method in the GARCH literature. In the univariate class, Geweke (1989) suggested the importance sampling technique for estimating ARCH models, while Bauwens and Lubrano (1998), Kim, Shephard and Chib (1998), Nakatsuma (2000), Vrontos, Dellaportas and Politis (2000), and Mitsui and Watanabe (2003) proposed several MCMC methods. Asai (2006) compared the above 
MCMC methods, and found that the best method is the so-called 'tailored' approach based on the acceptance-rejection Metropolis-Hastings (ARMH) algorithm, with respect to the mixing, efficiency and computational requirement. For multivariate GARCH models, Vrontos, Dellaportas and Politis (2003) used the Metropolis-Hastings algorithm of Vrontos, Dellaportas and Politis (2000), while Ishihara, Omori and Asai (2015) worked with the tailored ARMH algorithm.

Based on recent developments of the MCMC technique, we can use a fast and efficient approach, namely, the Delayed Rejection \& Adaptive Metropolis (DRAM) algorithm of Haario et al. (2006). The DRAM algorithm combines two ideas in the MCMC literature: adaptive Metropolis samplers (Haario et al. 1999, 2001) and delayed rejection (Tierney and Mira, 1999; Green and Mira, 2001; Mira 2002). The adaptive Metropolis sampler is based on the idea to create a Gaussian proposal distribution with a covariance matrix calibrated using the sample path of the MCMC chain. The basic idea of the delayed rejection is that, upon rejection in a Metropolis-Hastings algorithm, instead of advancing time and retaining the same position, a higher stage move is proposed to improve efficiency of the resulting MCMC estimators. We can apply the DRAM algorithm to various univariate and multivariate conditional volatility models.

We explain the delayed rejection and the adaptive Metropolis-Hastings algorithm of the DRAM algorithm of Haario et al. (2006). For the delayed rejection part, suppose the current position of the Markov chain is $\boldsymbol{\theta}_{n}=\theta$. As in a regular Metropolis-Hastings, a candidate move, $\boldsymbol{\theta}_{c}^{(1)}$, is generated from a candidate generating density, $q_{1}(\theta, \cdot)$ and accepted with the usual probability $\alpha_{1}\left(\theta, \theta_{c}^{(1)}\right)=\min \left(1, \frac{N_{1}}{D_{1}}\right)$, where $N_{1}=\pi\left(\theta_{c}^{(1)}\right) q_{1}\left(\theta_{c}^{(1)}, \theta\right)$ and $D_{1}=\pi(\theta) q_{1}\left(\theta, \theta_{c}^{(1)}\right)$ When it is rejected, instead of retaining the same position, $\boldsymbol{\theta}_{n+1}=\theta$, as we would do in a standard MetropolisHastings, a second stage move, $\boldsymbol{\theta}_{c}^{(1)}$, is proposed. The second stage proposal is allowed to depend not only on the current position of the chain but also on the rejected proposal: $q_{2}\left(\theta, \theta_{c}^{(1)}, \cdot\right)$. 
The second stage proposal is accepted with probability $\alpha_{2}\left(\theta, \theta_{c}^{(1)}, \theta_{c}^{(2)}\right)=\min \left(1, \frac{N_{2}}{D_{2}}\right)$, where $N_{2}=\pi\left(\theta_{c}^{(2)}\right) q_{1}\left(\theta_{c}^{(2)}, \theta_{c}^{(1)}\right) q_{2}\left(\theta_{c}^{(2)}, \theta_{c}^{(1)}, \theta\right)\left[1-\alpha_{1}\left(\theta_{c}^{(2)}, \theta_{c}^{(1)}\right)\right]$ and $D_{2}=\pi(\theta) q_{1}\left(\theta, \theta_{c}^{(1)}\right) q_{2}\left(\theta, \theta_{c}^{(1)}, \theta_{c}^{(2)}\right)[1-$ $\left.\alpha_{1}\left(\theta, \theta_{c}^{(1)}\right)\right]$. This process of delaying rejection can be iterated. If $q_{i}$ denotes the proposal density at the $i$-th stage, the acceptance probability at that stage is given by $\alpha_{i}\left(\theta, \theta_{c}^{(1)}, \ldots, \theta_{c}^{(i)}\right)=$ $\min \left(1, \frac{N_{i}}{D_{i}}\right)$, where

$$
\begin{aligned}
& D_{i}= q_{i}\left(\theta, \ldots, \theta_{c}^{(i)}\right)\left[q _ { i - 1 } ( \theta , \ldots , \theta _ { c } ^ { ( i - 1 ) } ) \left[q_{i-2}\left(\theta, \ldots, \theta_{c}^{(i-2)}\right) \cdots\right.\right. \\
& {\left.\left.\left[q_{2}\left(\theta, \theta_{c}^{(1)}, \theta_{c}^{(2)}\right)\left[q_{1}\left(\theta, \theta_{c}^{(1)}\right) \pi(\theta)-N_{1}\right]-N_{2}\right]-N_{3}\right] \cdots-N_{i-1}\right] } \\
& N_{i}=\pi\left(\theta_{c}^{(i)}\right) q_{1}\left(\theta_{c}^{(i)}, \theta_{c}^{(i-1)}\right) q_{2}\left(\left(\theta_{c}^{(i)}, \theta_{c}^{(i-1)}, \theta_{c}^{(i-2)}\right) \cdots q_{i}\left(\theta_{c}^{(i)}, \theta_{c}^{(i-1)}, \ldots, \theta\right)\right. \\
& \quad \times\left[1-\alpha_{1}\left(\theta_{c}^{(i)}, \theta_{c}^{(i-1)}\right)\right]\left[1-\alpha_{2}\left(\left(\theta_{c}^{(i)}, \theta_{c}^{(i-1)}, \theta_{c}^{(i-2)}\right)\right] \cdots\left[1-\alpha_{i-1}\left(\theta_{c}^{(i)}, \theta_{c}^{(i-1)}, \ldots, \theta_{c}^{(1)}\right)\right] .\right.
\end{aligned}
$$

As shown in Mira (2001), the process of delaying rejection can be interrupted at any stage. In our analysis, we try at most 5 times to move away from the current position, otherwise we let the chain stay where it is.

Now we turn to the explanation of the adaptive Metropolis-Hastings algorithm. The basic idea is to create a Gaussian proposal distribution with a covariance matrix calibrated using the sample path of the MCMC chain. Following Haario et al. (2006), when the current position is $\boldsymbol{\theta}_{n}=\theta_{n}$, we consider the proposal distribution with the multivariate normal distribution with mean $\theta$ and covariance matrix defined by:

$$
S_{n}= \begin{cases}S_{0}, & n \leq n_{0} \\ s_{p} \operatorname{Cov}\left(\theta_{0}, \ldots, \theta_{n}\right), & n>n_{0}\end{cases}
$$

where $s_{p}=2.4^{2} / p, p$ is the dimension of $\boldsymbol{\theta}$, and Cov creates the sample covariance matrix. In our analysis, we set $n_{0}=2,000$. Combining the two approaches, Haario et al. (2006) showed the ergodicity of the DRAM algorithm. 


\subsection{Bayesian Estimation of Realized MEGARCH Models}

Let $\boldsymbol{\theta}=\left(\operatorname{vech}(\boldsymbol{\Omega})^{\prime}, \operatorname{vech}(\boldsymbol{\Phi})^{\prime}, \boldsymbol{\gamma}^{\prime}, \operatorname{vech}(\boldsymbol{\Lambda})^{\prime}, \operatorname{vech}(\boldsymbol{\Xi})^{\prime}, \boldsymbol{\delta}^{\prime}, \operatorname{vech}(\boldsymbol{\Upsilon})^{\prime}, \operatorname{vech}(\boldsymbol{\Psi})^{\prime}, \operatorname{vech}(\boldsymbol{L})^{\prime}\right)^{\prime}$, where $\boldsymbol{L}$ is the lower triangular matrix calculated by the Cholesky decomposition of $\boldsymbol{\Sigma}_{u}$. Rather than sampling $\boldsymbol{\Sigma}_{u}$, we consider sampling $\boldsymbol{L}$ as this parametrization always guarantees the positive definiteness of $\boldsymbol{\Sigma}_{u}$ without imposing any restrictions. For $\boldsymbol{Y}=\left(\boldsymbol{y}_{1}, \ldots, \boldsymbol{y}_{T}\right)$ and $\boldsymbol{X}=\left(\boldsymbol{X}_{1}, \ldots, \boldsymbol{X}_{T}\right)$, the joint density function is given by:

$$
f(\boldsymbol{Y}, \boldsymbol{X} \mid \boldsymbol{\theta})=\prod_{t=1}^{T} f\left(\boldsymbol{y}_{t} \mid \boldsymbol{H}_{t}\right) f\left(\boldsymbol{X}_{t} \mid \boldsymbol{y}_{t}, \boldsymbol{H}_{t}, \boldsymbol{\theta}\right)
$$

with

$$
\begin{aligned}
& f\left(\boldsymbol{y}_{t} \mid \boldsymbol{H}_{t}\right) \propto\left|\operatorname{Exp}\left(\boldsymbol{H}_{t}\right)\right|^{-1 / 2} \exp \left(-\frac{1}{2} \boldsymbol{y}_{t}^{\prime} \operatorname{Exp}\left(-\boldsymbol{H}_{t}\right) \boldsymbol{y}_{t}\right), \\
& f\left(\boldsymbol{X}_{t} \mid \boldsymbol{y}_{t}, \boldsymbol{H}_{t}, \boldsymbol{\theta}\right) \propto\left|\boldsymbol{\Sigma}_{\boldsymbol{u}}\right|^{-1 / 2} \exp \left(-\frac{1}{2} \operatorname{vech}\left(\boldsymbol{U}_{t}\right)^{\prime} \boldsymbol{\Sigma}_{u}^{-1} \operatorname{vech}\left(\boldsymbol{U}_{t}\right)\right),
\end{aligned}
$$

where $\boldsymbol{U}_{t}=\boldsymbol{X}_{t}-\boldsymbol{\Xi}-\boldsymbol{H}_{t}-\boldsymbol{\Delta}\left(\operatorname{Exp}\left(-0.5 \boldsymbol{H}_{t}\right) \boldsymbol{y}_{t}\right)$ and $\boldsymbol{H}_{1}=\boldsymbol{\Omega}$.

We assume the multivariate (truncated) normal distribution for the prior distribution:

$$
\begin{aligned}
& \operatorname{vech}(\boldsymbol{\Omega}) \sim N\left(\boldsymbol{\mu}_{\omega}, \boldsymbol{\Sigma}_{\omega}\right), \quad \operatorname{vech}(\boldsymbol{\Phi}) \sim N\left(\boldsymbol{\mu}_{\phi}, \boldsymbol{\Sigma}_{\phi}\right) \times \boldsymbol{S}(\boldsymbol{\Phi}), \quad \gamma \sim N\left(\boldsymbol{\mu}_{\gamma}, \boldsymbol{\Sigma}_{\gamma}\right), \\
& \operatorname{vech}(\boldsymbol{\Lambda}) \sim N\left(\boldsymbol{\mu}_{\lambda}, \boldsymbol{\Sigma}_{\lambda}\right), \quad \operatorname{vech}(\boldsymbol{\Xi}) \sim N\left(\boldsymbol{\mu}_{\xi}, \boldsymbol{\Sigma}_{\xi}\right), \quad \boldsymbol{\delta} \sim N\left(\boldsymbol{\mu}_{\delta}, \boldsymbol{\Sigma}_{\delta}\right), \\
& \operatorname{vech}(\boldsymbol{\Upsilon}) \sim N\left(\boldsymbol{\mu}_{v}, \boldsymbol{\Sigma}_{v}\right), \quad \operatorname{vech}(\boldsymbol{\Psi}) \sim N\left(\boldsymbol{\mu}_{\psi}, \boldsymbol{\Sigma}_{\psi}\right), \quad \operatorname{vech}(\boldsymbol{L}) \sim N\left(\boldsymbol{\mu}_{l}, \boldsymbol{\Sigma}_{l}\right),
\end{aligned}
$$

where $\boldsymbol{S}(\boldsymbol{\Phi})$ is the region in which $\boldsymbol{\Phi}$ satisfies the stationary condition, $\mu_{\omega}=-4 \mathbf{1}_{m^{*}}, \boldsymbol{\Sigma}_{\omega}=5 I_{m^{*}}$, $\mu_{\phi}=\operatorname{vech}\left(0.15 I_{m}+0.8 \mathbf{1}_{m} \mathbf{1}_{m}^{\prime}\right), \boldsymbol{\Sigma}_{\phi}=5 I_{m^{*}}, \mu_{\gamma}=\mathbf{0}, \boldsymbol{\Sigma}_{\gamma}=5 I_{m}, \mu_{\lambda}=0.01 \mathbf{1}_{m^{*}}, \boldsymbol{\Sigma}_{\lambda}=5 I_{m^{*}}$, $\mu_{\xi}=\mathbf{0}, \boldsymbol{\Sigma}_{\xi}=5 I_{m^{*}}, \boldsymbol{\mu}_{\delta}=\mathbf{0}, \boldsymbol{\Sigma}_{\delta}=5 I_{m}, \mu_{v}=0.01 \mathbf{1}_{m^{*}}, \boldsymbol{\Sigma}_{v}=5 I_{m^{*}}, \mu_{\psi}=0.01 \mathbf{1}_{m^{*}}, \boldsymbol{\Sigma}_{\psi}=5 I_{m^{*}}$, and $\mathbf{1}_{m}$ is the $m$-dimensional vector of ones.

With the prior distribution $p(\boldsymbol{\theta})$, we consider the joint posterior distribution:

$$
\pi(\boldsymbol{\theta} \mid \boldsymbol{Y}, \boldsymbol{X}) \propto p(\boldsymbol{\theta}) f(\boldsymbol{Y}, \boldsymbol{X} \mid \boldsymbol{\theta}),
$$


which is proportional to the prior density times the joint density. To obtain the posterior quantities of the parameters from the posterior distribution, we implement the MCMC algorithm:

\section{Initialize $\boldsymbol{\theta}$.}

2. Generate $\boldsymbol{\theta} \mid \boldsymbol{Y}, \boldsymbol{X}$ by the DRAM algorithm.

3. Go to Step 2.

For the implementation issues, we discard the first 5,000 samples as the burn-in and use the subsequent 10,000 samples for estimation. We also set $S_{0}$ as the asymptotic covariance matrix of the ML estimates of the realized MEGARCH model, and draw an initial value of $\boldsymbol{\theta}$ from a multivariate normal distribution with the mean of their corresponding values of prior distribution and the covariance matrix, $S_{0}$.

\subsection{Illustration with Simulated Data}

We generated $T=1,000$ observations for the bivariate $(m=2)$ realized MEGARCH model $(2)$ (5), with parameter values listed in Table 1, which also shows the ML and Bayesian MCMC results. For the Bayesian MCMC results, the posterior means are computed by averaging the simulated draws; The $95 \%$ credible intervals are calculated using the 2.5 th and 97.5 th percentiles of the simulated draws. $P$-value is for the convergence diagnostics suggested by Geweke (1992), in which the test statistic approaches to the standard normal distribution if the corresponding Markov chain is stationary for the retained 10,000 samples. $P$-values indicate that all series converge after discarding the first 5,000 samples. Compared wth the ML estimates, the posterior means are close to the ML estimates. The $95 \%$ credible intervals are wider than the $95 \%$ confidence interval for the data set. The acceptance rate for the DRAM algorithm is 0.9435 , which is close to 1 due to the delayed rejection part of the DRAM. 
Figure 1 shows the estimated posterior densities for $\left(\omega_{11}, \omega_{21}, \omega_{22}, \phi_{11}, \phi_{21}, \phi_{22}\right)$. Except for $\phi_{11}$, the estimated desnities are far from the normal distribution with the same mean and variance. As shown by Vrontos et al. (2003) and Asai (2006), the posterior densities for the GARCH class model are generally non-normal.

Figure 2 illustrates the news impact curve, which shows effects from $i$ th return to the $(i, j)$ th element of the one-step ahead conditional covariance matrix $(i, j=1,2)$, given by equation $(7)$. As a feature of the MCMC technique, we obtain its posterior mean and 95\% interval simultaneously. For the specification with the data set, the effects from the $i$ th return $(i=1,2)$ on its own conditional variance draws asymmetric curves, while the effects on other conditional variance give (nearly) flat lines. The effects on conditional co-volatility give asymmetric curves, but they are negligible when the impacts are positive.

\section{Empirical Analysis}

Three stocks traded on the New York Stock Exchange are selected, namely: Bank of America (BAC), General Electric (GE), and International Business Machines (IBM). Based on the vector of returns for the $m=3$ stocks computed for a 1-minute interval of trading day at $t$ between 9:30 am and 4:00 pm, we calculated the daily values of the estimates of integrated co-volatility matrix, via the approach of Koike (2016). The estimator of Koike (2016) is robust to jumps and microstructure noise, and has an ability to handle asynchronicity of the times at which transactions are recorded. As in Asai and McAleer (2017), we modify the estimator of Koike (2016) to guarantee the positive definiteness of the covariance matrix, by the threshold method of Bickel and Levina (2008). We denote the estimate of integrated co-volatility matrix at dat $t$ as $\boldsymbol{C}_{t}$, giving $\boldsymbol{X}_{t}=\log \left(\boldsymbol{C}_{t}\right)$. The sample period starts at August 31, 2006, and ends on October 26, 
2012, giving 1500 observations. We also calculated the corresponding return vector, $\boldsymbol{y}_{t}$.

The sample is divided into two periods. The first 1000 observations cover the bankruptcy of Lehmann Brothers (September 15, 2008) and the Greek government requesting an initial loan from the EU (April 23, 2010). The former is the start of the global financial crisis, while the latter is the beginning of the European debt crisis. The last 500 observations are used for forecasting.

Table 2 presents the descriptive statistics of the returns, volatility ad co-volatility. The empirical distribution of the returns is heavily skewed to the left and is highly leptokurtic. Regarding volatility and co-volatility, they are skewed to the right, with evidence of heavy-tails in all series. Figure 3 shows the volatility and co-volatility of the three stocks, indicating that volatilities and co-volatilities are high in the period of turbulence caused by the global and European financial crises.

Using the return, volatility and co-volatility for three stocks, we estimated two kinds of realized MEGARCH models: one is based on the asymmetric function (5), while the other is based on (6). To compare these models, we also estimate two MEGARCH models (2) and (4), with $\boldsymbol{U}_{t}=O$ and $\boldsymbol{G}(\varepsilon)$ defined by (5) or (6), respectively. As a benchmark model, we use the BEKK model of Baba et al. (1987) and Engle and Kroner (1995):

$$
\begin{aligned}
\boldsymbol{y}_{t} & =\underline{\boldsymbol{H}}_{t}^{1 / 2} \varepsilon_{t}, \\
\underline{\boldsymbol{H}}_{t+1} & =\underline{\boldsymbol{\Omega}}+\underline{\boldsymbol{A}}\left(\boldsymbol{y}_{t} \boldsymbol{y}_{t}^{\prime}-\underline{\boldsymbol{\Omega}}\right) \underline{\boldsymbol{A}}^{\prime}+\underline{\boldsymbol{B}}\left(\underline{\boldsymbol{H}}_{t}-\underline{\boldsymbol{\Omega}}\right) \underline{\boldsymbol{B}}^{\prime},
\end{aligned}
$$

where $\underline{\boldsymbol{H}}_{t}$ is the conditional covariance matrix, $\underline{\boldsymbol{A}}$ and $\underline{\boldsymbol{B}}$ are $m \times m$ square matrices, $\underline{\boldsymbol{\Omega}}$ is an $m$-dimensional positive definite matrix. The specification is known as the 'variance targeting' BEKK model (see Laurent, Rombouts, and Violante (2012)).

Table 3 presents diagnostic results for the MCMC methods for estimating the five models. The acceptance rate is close to one for the delayed rejection of the DRAM algorithm. The minimum of 
thw $P$-values diagnostics suggested by Geweke (1992) indicates that all Markov chains converged.

To compare the in-sample performance of the five models, we use the DIC (deviance information criterion) of Spiegelhalter et al. (2002), defined by:

$$
\begin{aligned}
D I C & =E_{\left.\boldsymbol{\theta}\right|_{\boldsymbol{Y}, \boldsymbol{X}}}[D(\boldsymbol{\theta})]+p_{D}, \\
p_{D} & =E_{\left.\boldsymbol{\theta}\right|_{\boldsymbol{Y}, \boldsymbol{X}}}[D(\boldsymbol{\theta})]-D\left(E_{\boldsymbol{\theta} \mid \boldsymbol{Y}, \boldsymbol{X}}[\boldsymbol{\theta}]\right), \quad D(\boldsymbol{\theta})=-2 \log f(\boldsymbol{Y}, \boldsymbol{X} \mid \boldsymbol{\theta})+C_{\text {data }}
\end{aligned}
$$

where $C_{d a t a}$ is a constant term that depends only on the data set $\{\boldsymbol{Y}, \boldsymbol{X}\}$. Table 4 shows the estimates of DIC for the five models. The realized MEGARCH model (quadratic function) has the smallest DIC estimate, while the BEKK model has the largest DIC. Taking account of the minimum and maximum values in the fluctuations of DIC, there are clear differences between the realized MEGARCH models and the MEGACH models, and differences between the MEGARCH models and BEKK model. On the other hand, there is an overlap of the distributions of DICs of the two realized MEGACRH models. Compared with the differences in the models, the differences based on the asymmetric function are minor.

Figure 4 shows the box plots of the posterior densities for the parameters of the realized MEGARCH model with the quadratic function. Figure 4 implies that the posterior densities have skewed and heavy-tails, which are non-normal. The densities of $\phi_{i j}$ have a mass larger than 0.9 , indicating high persistence for the process of $\boldsymbol{H}_{t}$. To examine the relationship between returns and future (co-)volatilities, we need a careful consideration since the $(i, j)$-element of $\boldsymbol{H}_{t}$ does not necessarily correspond to the $(i, j)$-element of $\operatorname{Exp}\left(\boldsymbol{H}_{t}\right)$. Figure 5 presents the news impact curves for the realized MEGARCH models with the quadratic function. Figure 5(a)(j)(r) shows the asymmetric news impacts for their own future volatilities. Figure $5(\mathrm{~g})(\mathrm{m})$ indicates that the 
news impacts from GE/IBM to future volatility of BAC are relatively large, while Figure $5(\mathrm{~h})(\mathrm{l})$ illustrates the asymmetric effects from GE/IBM to future co-volatility between BAC and itself. The remaining news effects are relatively small. The results are reasonable as the financial sector is considered to be sensitive to the fluctuations of stocks in other sectors for the period which covers the GFC and EDC.

Fixing the sample size as $T=1,000$, we re-estimated all the models to obtain one-step-ahead forecasts of co-volatility matrices based on updated posterior distributions. We use $\boldsymbol{C}_{h}$ as a proxy for the true co-volatility matrix, and define the forecast error matrix as:

$$
\boldsymbol{E}_{h}=\boldsymbol{C}_{h}-\operatorname{Exp}\left(\boldsymbol{H}_{h}^{f}\right)
$$

where $\boldsymbol{H}_{h}^{f}$ is the one-step-ahead forecast at time $h$. We compare the five models' out-of-sample forecast root-mean-squared error (RMSE) based on the Frobenius norm of the forecast error, which is defined by

$$
F N=\frac{1}{500} \sum_{h}\left\|\boldsymbol{E}_{T+h}\right\|=\frac{1}{500} \sum_{t}\left[\sum_{i, j} e_{i j, T+h}^{2}\right]^{1 / 2},
$$

for the last 500 observations.

Table 5 presents the estimates of $F N$. The realized MEGACRH model (quadratic function) has the smallest posterior mean, which is close to the results of the alternative realized MEGARCH model. The upper bounds of the $95 \%$ credible interval of the $\mathrm{FN}$ of the realized MEGARCH models are smaller than the lower bounds for the BEKK and the two MEGARCH models, implying the superiority of the structure of the realized MEGARCH model. Figure 6 shows the forecasts of the dynamic correlations and their corresponding realized measures calculated via $c_{i j, t} / \sqrt{x_{i i, t} c_{j j, t}}$, where $c_{i j, t}$ is the $(i, j)$ element of $\boldsymbol{C}_{t}$. Figure 6 indicates that the forecasts of the correlation dynamics capture the realized correlation well. 
For the period which covers the GFC and EDC, the empirical results show that the new realized MEGARCH model gives the best fit to the data, and the best forecasts. Between the two realized MEGARCH models, the quadratic asymmetric function provides better results, although the difference is minor.

\section{Conclusion}

The paper developed a new realized matrix-exponential GARCH (MEGARCH) model, which is an extension of univariate realized exponential GARCH model of Hansen and Huang (2016). We considered the Bayesian MCMC estimation technique, which gives non-normal posterior distributions. Using returns and realized measures of the co-volatility matrix for three stocks traded on NYSE, we found that the realized MEGARCH models outperformed the BEKK and MEGARCH models for in-sample and out-of-sample performance. The news impact curves based on the posterior densities presented reasonable results.

The new realized MEGARCH model and Bayesian MCMC estimation open the possibility for many interesting research directions. We may develop a realized version of the BEKK model, the dynamic conditional correlation model of Engle (2002), and the Cholesky GARCH model of Dellaportas and Pourahmadi (2012). For these issues, we need to wait for further research on modeling the appropriate specification of the measurement equation of the realized measure of co-volatility matrix, so that its conditional and realized covariance matrices are positive definite. 


\section{References}

Asai, M. (2006), "Comparison of MCMC methods for estimating GARCH models", Journal of Japan Statistical Society, 36,199-212.

Asai, M. and M. McAleer (2017), "The impact of jumps and leverage in forecasting co-volatility", Econometric Reviews, 36, 638-650.

Baba, Y., Engle, R., Kraft, D. and Kroner, K. (1987), "Multivariate simultaneous generalized ARCH", Unpublished Paper, University of California, San Diego.

Bauwens, L., and Lubrano, M. (1998), "Bayesian inference on GARCH models using the Gibbs sampler," Econometrics Journal, 1, c23-c46.

Bickel, P. J., and E. Levina (2008), "Covariance regularization by thresholding", Annals of Statistics, 36, $2577-2604$.

Chiu, T.Y.M., T. Leonard, and K.-W. Tsui (1996), "The matrix-logarithmic covariance model", Journal of the American Statistical Association, 91, 198-210.

Dellaportas, P. and M. Pourahmadi (2012), "Cholesky-GARCH models with applications to finance", Statistics and Computing, 22, 849-855

Engle, R.F. (2002), "Dynamic conditional correlation: A simple class of multivariate generalized autoregressive conditional heteroskedasticity models", Journal of Business 83 Economic Statistics, 20, $339-350$.

Engle, R.F., and G. Gallo (2006), "A multiple indicators model for volatility using intra-daily data", Journal of Econometrics, 131, 3-27.

Engle, R.F. and Kroner, K. F. (1995), "Multivariate simultaneous generalized ARCH", Econometric Theory, 11, 122-150.

Engle, R.F., and V. Ng (1993), "Measuring and testing the impact of news on volatility", Journal of Finance, 48, 1749-1778.

Geweke, J. (1989), "Exact predictive density for linear models with ARCH disturbances", Journal of Econometrics, 40, 63-86.

Geweke, J. (1992), "Evaluating the accuracy of sampling-based approaches to the calculation of posterior moments", in Bayesian Statistics 4 (eds. J.M. Bernardo, J.O. Berger, A.P. David and A.F.M. Smith), pp. 169-193, Oxford University Press, Oxford.

Green, P.J. and A. Mira (2001), "Delayed rejection in reversible jump Metropolis-Hastings", Biometrika, 88, 1035-1053.

Haario, H., M. Laine, A. Mira, and E. Saksman (2006), "DRAM: Efficient adaptive MCMC", Statistics and Computing, 16, 339-354.

Haario H., E. Saksman, and J. Tamminen (1999), "Adaptive proposal distribution for random walk Metropolis algorithm", Computational Statistics, 14, 375-395.

Haario H., E. Saksman, and J. Tamminen (2001), "An adaptive Metropolis algorithm", Bernoulli, 7, 223-242.

Hansen, P.R. and Z. Huang (2016), "Exponential GARCH modeling with realized measures of volatility", Journal of Business \&ु Economic Statistics, 34, 269-287. 
Hansen, P.R., Z. Huang, and H.H. Shek (2012), "Realized GARCH: A complete model of returns and realized measures of volatility", Journal of Applied Econometrics, 27, 877-906.

Ishihara, T., Y. Omori and M. Asai (2016), "Matrix exponential stochastic volatility with cross leverage", Computational Statistics \& Data Analysis, 100, 331-350.

Kawakatsu, H. (2005), "Matrix exponential GARCH", Journal of Econometrics, 134, 95-128.

Kim, S., Shephard, N., and Chib, S. (1998), "Stochastic volatility: Likelihood inference and comparison with ARCH models", Review of Economic Studies, 65, 361-393.

Koike, Y.(2016), "Estimation of integrated covariances in the simultaneous presence of non-synchronicity, microstructure noise and jumps", Econometric Theory, 32, 533-611.

Laurent, S., J.V.K. Rombouts and F. Violante (2012), "On the forecasting accuracy of multivariate GARCH models", Journal of Applied Econometrics, 27, 934-955.

McAleer, M. (2005), "Automated inference and learning in modeling financial volatility", Econometric Theory, 21, 232-261.

Mira A. (2001), "On Metropolis-Hastings algorithms with delayed rejection”, Metron, 59, 231-241.

Mitsui, H., and T. Watanabe (2003), "Bayesian analysis of GARCH option pricing models," (in Japanese), the Journal of the Japan Statistical Society (Japanese Issue), 33, 307-324.

Nakatsuma, T. (2000), "Bayesian analysis of ARMA-GARCH models: A Markov chain sampling approach," Journal of Econometrics, 95, 57-69.

Nelson, D.B. (1991), "Conditional heteroskedasticity in asset returns: A new approach", Econometrica, $\mathbf{5 9}, 347-370$.

Shephard, N, and K. Sheppard (2010), "Realising the future: Forecasting with high frequency based volatility (HEAVY) models", Journal of Applied Econometrics, 25, 197-231.

Spiegelhalter, D.J., N.G. Best, B.P. Carlin, and A. van der Linde (2002), "Bayesian measures of model complexity and fit" (with discussion), Journal of the Royal Statistical Society, Series B, 64, 583-639.

Tierney L. and A. Mira (1999), "Some adaptive Monte Carlo methods for Bayesian inference", Statistics in Medicine, 18, 2507-2515.

Vrontos, I.D., Dellaportas, P., and Politis, D. (2000), "Full Bayesian inference for GARCH and EGARCH models," Journal of Business and Economic Statistics, 18, 187-198.

Vrontos, I. D., Dellaportas, P. and Politis, D. N. (2003), "Inference for some multivariate ARCH and GARCH models", Journal of Forecasting, 22, 427-446. 
Table 1: Comparison of ML and Bayesian MCMC Results for Simulated Data

\begin{tabular}{|c|c|c|c|c|c|}
\hline $\begin{array}{l}\text { Parameter } \\
\text { Value }\end{array}$ & $\begin{array}{c}\text { ML } \\
\text { Estimate }\end{array}$ & $\begin{array}{l}95 \% \text { Confidence } \\
\text { Interval }\end{array}$ & $\begin{array}{c}\text { Posterior } \\
\text { Mean }\end{array}$ & $\begin{array}{l}95 \% \text { Credible } \\
\text { Interval }\end{array}$ & $\begin{array}{l}P \text {-value } \\
(\text { Conv. })\end{array}$ \\
\hline$\omega_{11}=1$ & 1.1521 & $(0.7930,1.5112)$ & 1.2743 & $(0.3371,2.6156)$ & 0.9185 \\
\hline$\omega_{21}=0$ & 0.1850 & $(0.0289,0.3411)$ & 0.1915 & $(-0.1830,0.5547)$ & 0.8065 \\
\hline$\omega_{22}=1$ & 1.6565 & $(1.3510,1.9621)$ & 1.8296 & $(0.9419,2.6976)$ & 0.9612 \\
\hline$\phi_{11}=0.98$ & 0.9579 & $(0.9522,0.9636)$ & 0.9610 & $(0.9371,0.9830)$ & 0.9972 \\
\hline$\phi_{21}=0.80$ & 0.8098 & $(0.7963,0.8232)$ & 0.8068 & $(0.7563,0.8464)$ & 0.9980 \\
\hline$\phi_{22}=0.95$ & 0.9443 & $(0.9355,0.9532)$ & 0.9503 & $(0.9227,0.9805)$ & 0.9996 \\
\hline$\gamma_{1}=-0.4$ & -0.2482 & $(-0.2620,-0.2344)$ & -0.2550 & $(-0.2978,-0.2083)$ & 0.9830 \\
\hline$\gamma_{2}=-0.2$ & -0.2135 & $(-0.2235,-0.2035)$ & -0.2142 & $(-0.2447,-0.1817)$ & 0.9566 \\
\hline$\lambda_{11}=0.4$ & 0.3406 & $(0.3174,0.3638)$ & 0.3706 & $(0.2934,0.4377)$ & 0.9624 \\
\hline$\lambda_{21}=0.3$ & 0.1703 & $(0.1554,0.1851)$ & 0.1963 & $(0.1434,0.2495)$ & 0.9236 \\
\hline$\lambda_{22}=0.2$ & 0.0889 & $(0.0827,0.0952)$ & 0.0934 & $(0.0740,0.1156)$ & 0.9018 \\
\hline$\xi_{11}=-1.0$ & -1.4393 & $(-1.5255,-1.3531)$ & -1.5320 & $(-1.7445,-1.3128)$ & 0.9789 \\
\hline$\xi_{21}=-0.5$ & -0.6166 & $(-0.6459,-0.5872)$ & -0.6234 & $(-0.6965,-0.5408)$ & 0.9878 \\
\hline$\xi_{22}=-1.5$ & -2.1303 & $(-2.1945,-2.0662)$ & -2.1929 & $(-2.4124,-1.9637)$ & 0.9567 \\
\hline$\delta_{1}=-0.1$ & -0.0856 & $(-0.0978,-0.0735)$ & -0.0947 & $(-0.1303,-0.0555)$ & 0.9865 \\
\hline$\delta_{1}=-0.2$ & -0.2106 & $(-0.2211,-0.2001)$ & -0.2138 & $(-0.2543,-0.1763)$ & 0.9695 \\
\hline$v_{11}=0.20$ & 0.1700 & $(0.1480,0.1920)$ & 0.1930 & $(0.1136,0.2524)$ & 0.9234 \\
\hline$v_{21}=0.15$ & 0.0971 & $(0.0852,0.1090)$ & 0.1194 & $(0.0728,0.1650)$ & 0.9028 \\
\hline$v_{22}=0.10$ & 0.0371 & $(0.0317,0.0426)$ & 0.0352 & $(0.0066,0.0659)$ & 0.8096 \\
\hline$\psi_{11}=0.1$ & 0.4361 & $(0.4188,0.4534)$ & 0.4421 & $(0.3471,0.5010)$ & 0.9631 \\
\hline$\psi_{21}=0.3$ & 1.0591 & $(1.0446,1.0735)$ & 1.0598 & $(1.0129,1.1046)$ & 0.9990 \\
\hline$\psi_{22}=0.2$ & 0.4798 & $(0.4523,0.5073)$ & 0.4827 & $(0.3891,0.5837)$ & 0.9839 \\
\hline$l_{11}=0.500$ & 0.9144 & $(0.8811,0.9478)$ & 0.9404 & $(0.8262,1.0736)$ & 0.9985 \\
\hline$l_{21}=0.075$ & 0.0666 & $(0.0367,0.0965)$ & 0.0919 & $(0.0018,0.1845)$ & 0.7750 \\
\hline$l_{31}=-0.060$ & -0.0734 & $(-0.1055,-0.0412)$ & -0.0594 & $(-0.1590,-0.0326)$ & 0.7154 \\
\hline$l_{22}=0.250$ & 0.4268 & $(0.4078,0.4457)$ & 0.4307 & $(0.3900,0.4748)$ & 0.9686 \\
\hline$l_{32}=0.120$ & 0.1558 & $(0.1302,0.1814)$ & 0.1640 & $(0.0852,0.2535)$ & 0.8600 \\
\hline$l_{33}=0.200$ & 0.4097 & $(0.3950,0.4243)$ & 0.4286 & $(0.3810,0.4753)$ & 0.9960 \\
\hline Acceptance & & & & & \\
\hline Rate & & & 0.9435 & & \\
\hline
\end{tabular}

Note: $P$-value is for the convergence diagnostic statistics suggested by Geweke (1992). 
Table 2: Descriptive Statistics of Returns, Volatilities and Co-Volatilities

\begin{tabular}{c|rrcc}
\hline \hline Stock & \multicolumn{1}{c}{ Mean } & Std.Dev. & Skew. & Kurt. \\
\hline Return & & & & \\
BAC & -0.2423 & 3.6214 & -0.2525 & 13.054 \\
GE & 0.1230 & 1.2211 & -0.3447 & 7.1232 \\
IBM & -0.0511 & 1.8591 & -0.1674 & 11.200 \\
Volatility & & & & \\
BAC & 4.1286 & 10.338 & 8.8001 & 134.34 \\
GE & 0.7827 & 1.7139 & 8.4851 & 107.38 \\
IBM & 1.7100 & 4.0020 & 7.8978 & 92.847 \\
Co-Volatility & & & & \\
(BAC,GE) & 0.3694 & 1.2924 & 9.0682 & 124.53 \\
(BAC,IBM) & 0.6565 & 2.3697 & 7.9425 & 89.802 \\
(IBM,GE) & 0.4781 & 1.1807 & 7.5428 & 80.038 \\
\hline
\end{tabular}

Table 3: MCMC Diagnostic Results

\begin{tabular}{l|cccc}
\hline \hline Model & AR & Min. $P$-value & Median $P$-value & Max. P-value \\
\hline Real MEGARCH (qd) & 1.0000 & 0.1629 & 09345 & 0.9996 \\
Real MEGARCH (abs) & 1.0000 & 0.1624 & 0.8681 & 1.0000 \\
MEGARCH (qd) & 1.0000 & 0.1767 & 0.8600 & 0.9996 \\
MEGARCH (abs) & 0.9697 & 0.1765 & 0.7521 & 0.9920 \\
BEKK & 1.0000 & 0.1710 & 0.8821 & 0.9965 \\
\hline
\end{tabular}

Note: 'AR' denotes the acceptance rate of the DRAM algorithm. $P$-value is for the convergence diagnostic statistic of Geweke (1992). 
Table 4: DIC Estimates

\begin{tabular}{l|ccccc}
\hline \hline Model & Ranking & DIC & S.E. & DIC $_{\max }$ & DIC $_{\min }$ \\
\hline Real MEGARCH (qd) & 1 & 25776.56 & $(155.84)$ & 26278.34 & 25293.42 \\
Real MEGARCH (abs) & 2 & 26246.57 & $(334.48)$ & 27648.75 & 25148.74 \\
MEGARCH (qd) & 3 & 32414.06 & $(53.14)$ & 32677.01 & 32292.61 \\
MEGARCH (abs) & 4 & 32706.78 & $(150.76)$ & 33252.62 & 32287.65 \\
BEKK & 5 & 36373.84 & $(67.64)$ & 36679.49 & 36217.02 \\
\hline
\end{tabular}

Table 5: Estimates of Frovenious Norm for Out-of-Sample Forecast Errors

\begin{tabular}{l|ccc}
\hline \hline & & Posterior & $95 \%$ Credible \\
Model & Ranking & Mean & Interval \\
\hline Real MEGARCH (qd) & 1 & 5.9444 & $(5.8446,6.1054)$ \\
Real MEGARCH (abs) & 2 & 6.1601 & $(6.0535,6.3466)$ \\
MEGARCH (qd) & 3 & 7.3730 & $(7.2344,7.6333)$ \\
MEGARCH (abs) & 4 & 9.8408 & $(9.6167,10.6414)$ \\
BEKK & 5 & 10.0824 & $(9.8822,10.4021)$ \\
\hline
\end{tabular}


Figure 1: Estimated Posterior Density for Simulated Data
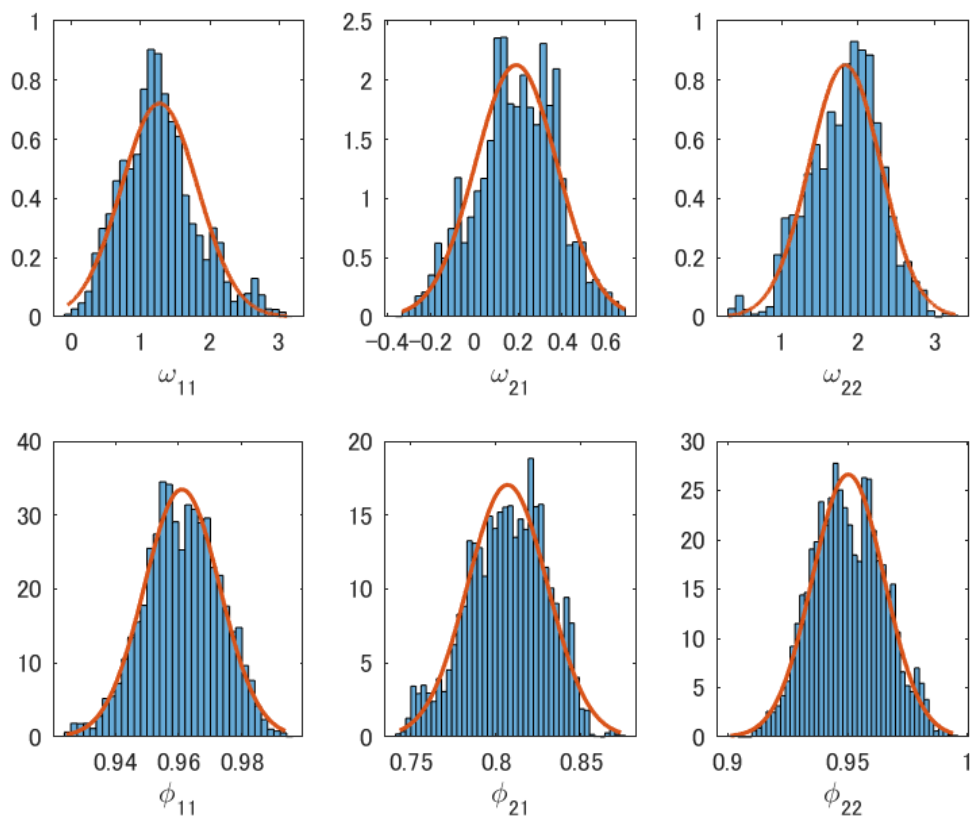

Note: Histograms are normalized to estimate densities. The red line shows the corresponding normal density with the same mean and variance. 
Figure 2: Estimated News Impact Curves for Simulated Data

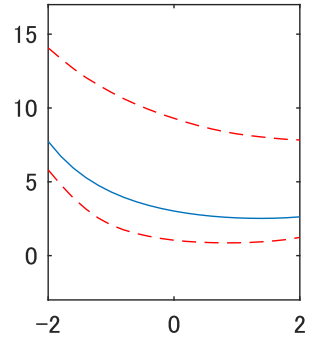

(a) $y_{1}$ to $v_{11}$

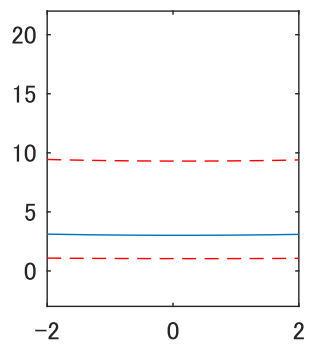

(d) $\mathrm{y}_{2}$ to $\mathrm{v}_{11}$

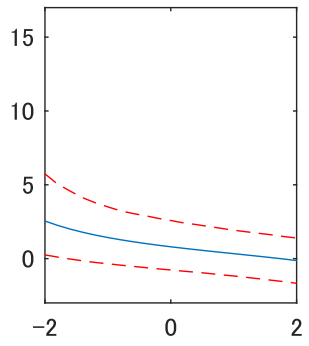

(b) $y_{1}$ to $v_{21}$

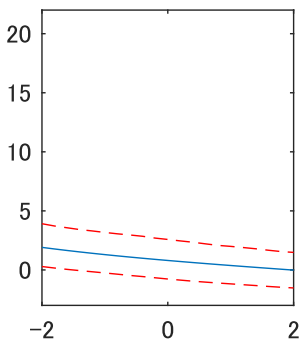

(e) $y_{2}$ to $v_{21}$

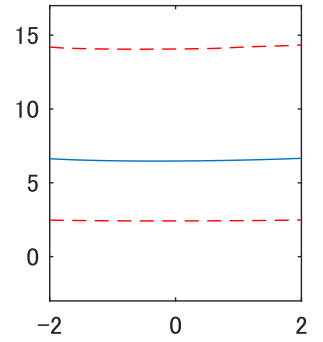

(c) $y_{1}$ to $v_{22}$

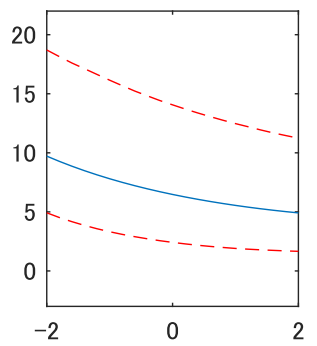

(f) $y_{2}$ to $v_{22}$

Note: Posterior mean (solid line) and 95\% interval (dotted lines) of the news impact curve via equation (7). 
Figure 3: Estimated Co-Volatility Matrix for Stock Returns
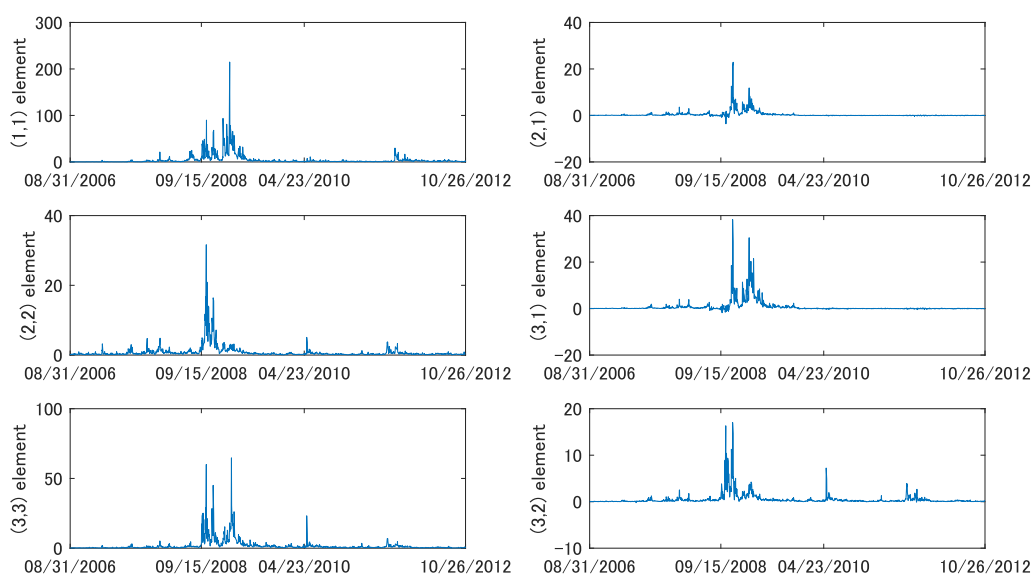

Note: Time series plots of $(i, j)$ th element of covariance matrix; BAC $(i, j=1)$, GE $(i, j=2)$ and $\operatorname{IBM}(i, j=3)$. 
Figure 4: Boxplots of Posterior Samples for the Realized MEGARCH Model
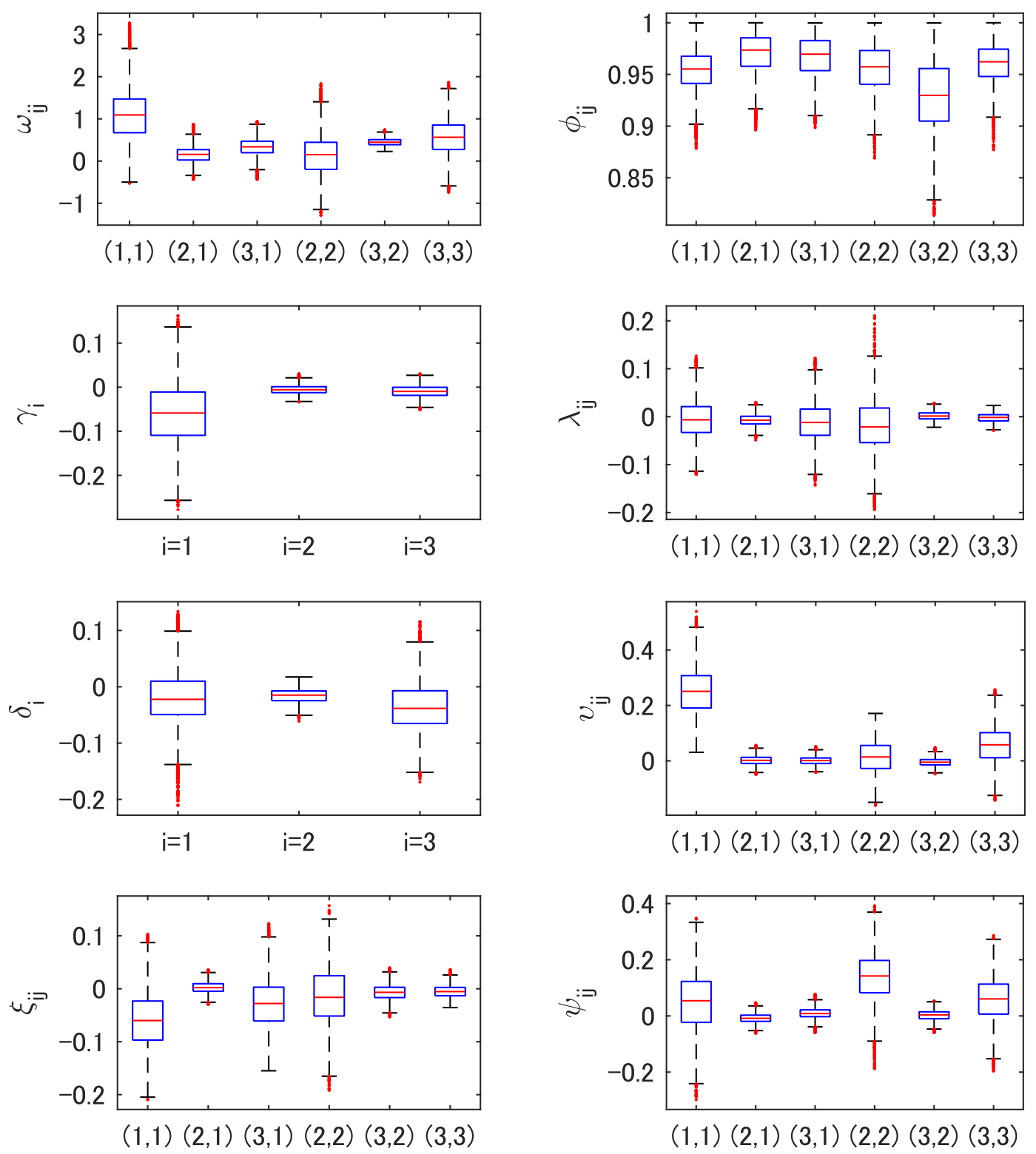

Note: The numbers for $i$ and $j$ indicate one of three stocks, namely, (1) BAC, (2) GE, and (3) IBM. 
Figure 5: Estimated News Impact Curves for Stock Market Data
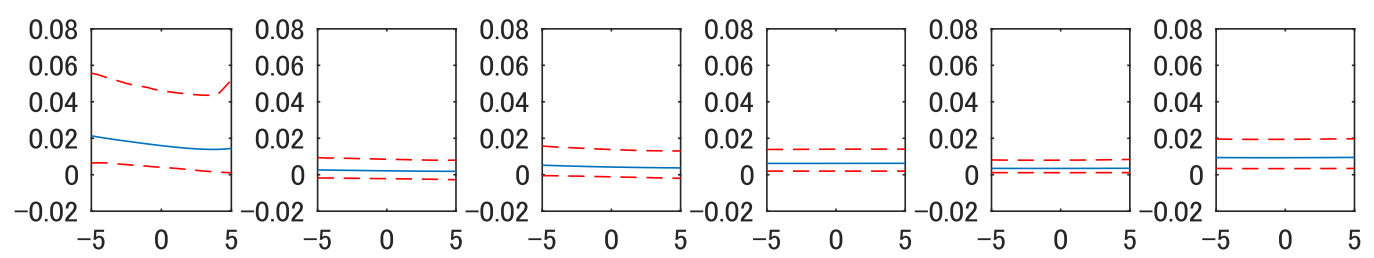
(a) $y_{1}$ to $v_{11}$
(b) $y_{1}$ to $v_{21}$
(c) $y_{1}$ to $v_{31}$

(d) $y_{1}$ to $v_{22}$

(e) $y_{1}$ to $v_{32}$

(f) $y_{1}$ to $v_{33}$
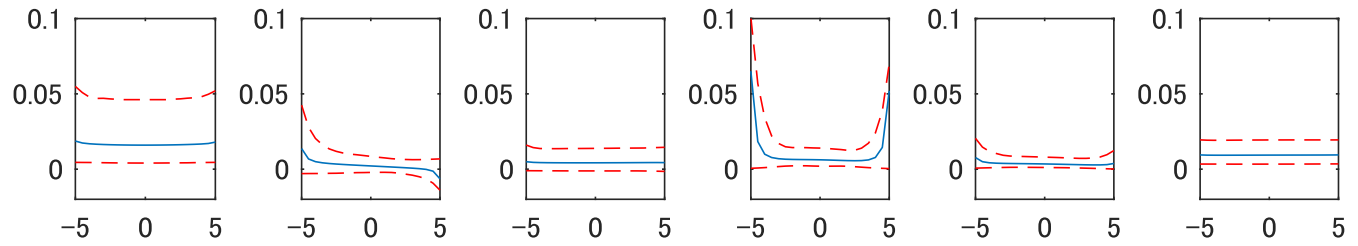

(g) $y_{2}$ to $v_{11}$

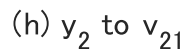

(i) $y_{2}$ to $v_{31}$

(j) $y_{2}$ to $v_{22}$

(k) $y_{2}$ to $v_{32}$

(I) $y_{2}$ to $v_{33}$
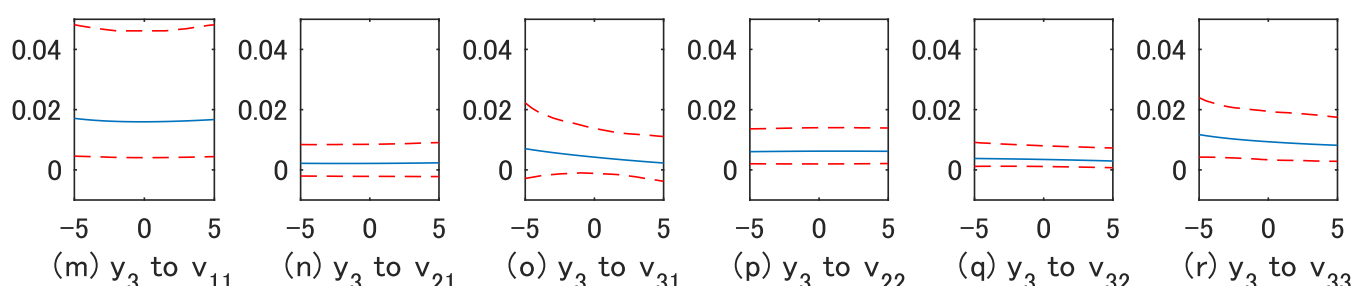

Note: Posterior mean (solid line) and 95\% interval (dotted lines) of the news impact curve via equation (7), which measures the effect on $(i, j)$ th element of co-volatility matrix by the impact of $k$ th return, where the number indicates three stocks, namely, (1) BAC, (2) GE, and (3) IBM. 
Figure 6: Realized Measures and Forecasts of Dynamic Correlations
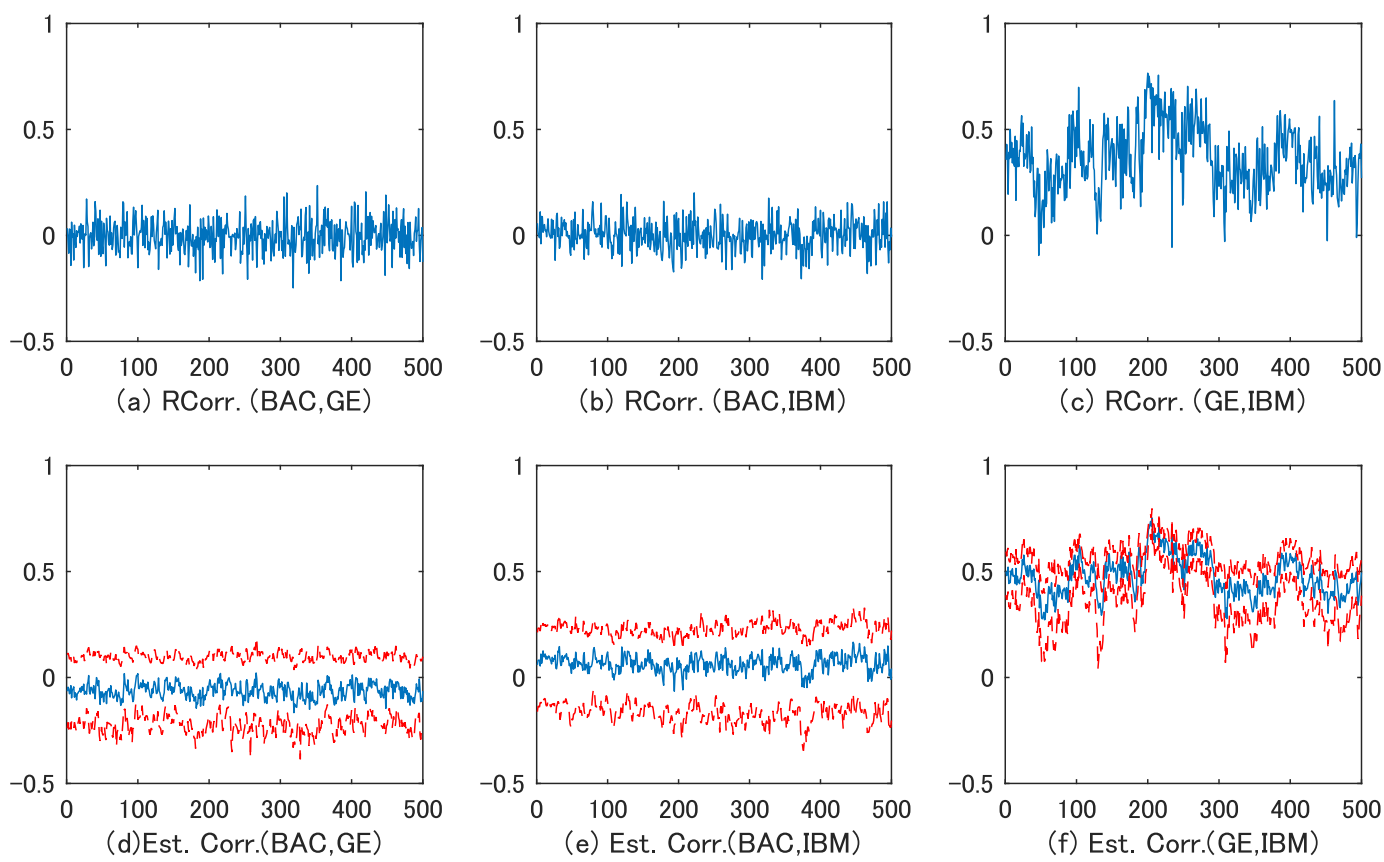

Note: Figures 6(a)-(c) show the realized correlations, while Figures 6(d)-(f) illustrate the estimated posterior means (solid line) and $95 \%$ intervals (dotted lines) of the one-step-ahead forecasts of correlation dynamics. 